\title{
"Slow" Metastable Decomposition of Oligosaccharide Cations Produced in an External Source Fourier Transform Mass Spectrometer
}

\author{
Lambert Ngoka and Carlito B. Lebrilla \\ Department of Chemistry, University of California, Davis, California, USA
}

\begin{abstract}
Unimolecular metastable decomposition of a-cyclodextrin cations is observed in a fast atom bombardment Fourier transform mass spectrometry instrument. Cleavage reactions occur at the glycosidic bonds to produce oligomeric fragment ions. The first-order rate constant for the decay of the protonated parent was measured and found to be $(4.2 \pm 1.0) \times 10^{2} \mathrm{~s}^{-1}$. (J Am Soc Mass Spectrom 1993, 4, 210-215)
\end{abstract}

A $s$ the current capability in mass spectrometry extends to still larger masses (i.e., $>10^{4}$ Dal.), the need arises to determine the dynamics of energy randomization after ion formation. Particularly for organic macromolecules, energy randomization is important for optimizing the production of structurally relevant fragment ions. The investigation of the unimolecular decomposition of ions continues to be a subject of considerable interest [1-22]. For a given internal energy, statistical theories, such as quasi-equilibrium theory [23, 24] and Rice, Ramsberger, Kassel, Marcus theory (RRKM) [2, 12] suggest a general decrease in the rates of various fragmentation pathways as the size of the molecule is increased. This trend is supported by the report of Demirev et al. [13] on the fragmentation of high-mass $(m / z 500-5800)$ ions that show's metastable decay occurring beyond $0.4 \mathrm{~ms}$ for ions produced by plasma desorption and fast-atom bombardment (FAB) $[25,26]$. Unimolecular rate constants as low as $2.3 \times 10^{3} \mathrm{~s}^{-1}$ are reported by Schueler et al. [4] with para-substituted phenylalanine ions produced by liquid secondary ion mass spectrometry or, more conveniently, FAB, and observed with a time-offlight mass spectrometer [4].

Metastable unimolecular decomposition of ions produced by direct ionization (e.g., electron impact) in Fourier transform mass spectrometry (FTMS) is not observed because metastable decay rates are generally faster than the millisecond timescale of FTMS. To observe unimolecular decay of ions produced in the FTMS instrument, it must be at least an order of

Address reprint requests to Carlito B. Lebrilla, Department of Chemistry, University of California, Davis, CA 95616. magnitude less than those reported by Schueler et al. [4]. Recently, Guan and Mclver [27] reported slow metastable decay of larger FAB-produced peptides in an external source FTMS instrument; however, actual unimolecular decomposition rate constants were not obtained. Slow metastable decay rates are of further interest because they are in the proper time regime to allow radiative cooling to be in competition with fragmentation reactions $[3,28]$. For example, radiative rates of $0.98 \times 10^{2} \mathrm{~s}^{-1}$ have been reported by McMahon and Kofel [3] for the protonated acetone dimer produced by collisional association in a FTMS instrument. This means that some of the ions trapped in the analyzer cell with sufficient energy to undergo metastable decay may instead remain intact by undergoing radiative decay.

Oligosaccharides are of particular interest in mass spectrometry because of their importance in several key biological processes. Difficulties arise in obtaining structurally relevant fragment ions in the $\mathrm{FAB} /$ mass spectrometry (MS) spectra. We previously reported differences between FAB/FTMS and TAB/MS (sector) spectra of oligosaccharides [29]. Fragment ion abundances are often greater in spectra obtained by FTMS than by sector mass spectrumetry [29]. These differences can be explained by the presence of slow metastable decay. Unimolecular metastable decay is readily observed in sector and TOF instruments; however, owing to the complicated nature of the ion motion and the mechanism for ion trapping, it is more difficult to observe metastable decay in the FTMS instrument. In this report, evidence is presented for the slow metastable decomposition of oligosaccharide ions produced by FAB ionization in an external source FTMS instrument. 


\section{Experimental}

Experiments were performed in a quadrupole FTMS [30-32] instrument built in our laboratory [29, 32, 33]. The instrument contains an external ion source and a quadrupole ion guide (radiofrequency only) to transport ions from the source into the analyzer cell. The $5-\mathrm{cm}$ stainless steel cubic analyzer cell is located in a homogeneous region of a 3-T superconducting magnet. Two stages of differential pumping between the source and the analyzer chambers allowed us to maintain pressures in the mid-10 $10^{-10}$-torr range in the analyzer chamber, even when sample was in place in the external source. The low background pressure precludes collisionally activated dissociation (CAD) of the trapped ions. Ions transported from the source through the quadrupole ion guide and into the analyzer cell do not pass through grids as in other external source FTMS designs, which could produce surface-induced dissociation [34]. Ions are extracted through a $1.5-\mathrm{mm}$ acceleration aperture and enter a 3.2-mm aperture in the front trapping plate of the analyzer cell.

All organic samples and substrates are commercially available (Sigma Chemical Co., St. Louis, $-\mathrm{MO}$ ). Typical samples are obtained by dissolving 1-2 $\mathrm{mg}$ of oligosaccharides in $0.5 \mathrm{~mL}$ of deionized water. Frnm this solution a $5-\mu \mathrm{L}$ aliquot is placed on the matrix material (1:1 glycerol:thioglycerol) on the surface of a copper probe tip. Protonated oligosaccharides are produced by bombarding the matrix solution containing the oligosaccharide with a $10-\mathrm{kV} \mathrm{Cs}^{+}$beam. Alkalated oligosaccharide ions are produced by doping the oligosaccharide solution with the appropriate metal salt solution.

The pulse scquence used for all of the experiments reported is shown in Figure 1. To observe unimolecular decay, a short ion injection time of $10 \mathrm{~ms}$ is used. The injection period involves the simultaneous ion formation (activation of cesium gun) and ion transmission (activation of quadrupole ion guide). The detection delay time, defined from the end of the injection pulse, is variable. To obtain reproducible signal intensities, experiments are performed within the first 3-5 min of sample introduction. This ensures that the concentration of analyte species on the probe remains relatively constant. Experiments are also duplicated in random time order to eliminate the effects of decreasing analyte concentration. This procedure did not affect the rate constants obtained. Because the metastable process occurs rapidly compared with the time scale of FTMS, experiments involving ion sweep or collisional cooling are not performed. These experiments would undoubtedly be useful in unambiguously determining the products of the unimolecular decomposition.

Ions that are already trapped are presumably undergoing metastable decay during the entire ion formation/injection period. Even shorter injection times would be preferable to minimize the amount of metastable decay during ion formation and transport.

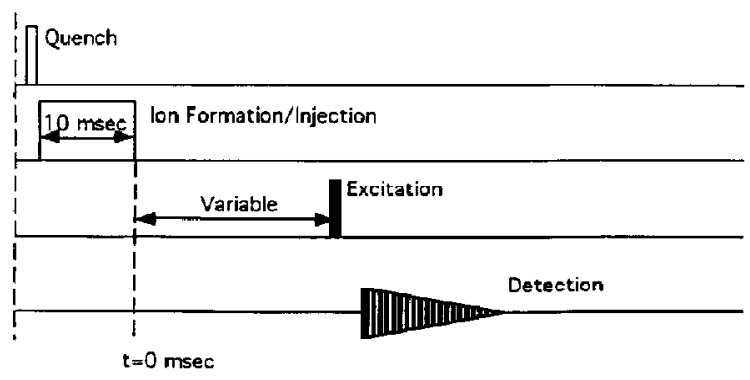

Figure 1. The pulse sequence used to investigate unimolecular metastable decay in the external source FTMS instrument. The ion formation pulse simultaneously turns on the cesium ion gun and the quadrupole ion guide during a period lasting $10 \mathrm{~ms}$. The detection delay times reported in the text are relative to the end of the ion formation pulse. Between 50 and 100 scans are typically accumulated for each spectrum.

The 10 -ms period is a compromise between obtaining sufficient signal to noise for analysis and observing the decay of the parent ion.

\section{Results}

The positive ion mass spectrum of $\alpha$-cyclodextrin is distinctive, with every polymeric sequence from the disaccharide fragment $(m / z 325)$ to the hexasaccharide (protonated parent ion, $\mathrm{m} / z$ 973) clearly observed (Figure 2, top). Fragmentation products are due to clcavages exclusivcly along the glycosidic bonds and correspond to masses of $m / z 325,487,649,811$, and $973\left([\mathrm{M}+\mathrm{H}]^{+}\right)$. The spectra are obtained in the lower resolution broad band mode to take advantage of the rapid, simultaneous detection feature of the instrument. To produce these spectra, 100 scans are accumulated and averaged.

When the detection delay time is lengthened, the intensity of the parent ion and the $m / z 811$ fragment decrease. Figure 2 illustrates a series of spectra with varying detection delay times of $1,3,5$, and $10 \mathrm{~ms}$. The intensity of the protonated parent decreases from the initial value of $73 \%$ of the base peak at $1 \mathrm{~ms}$ (Figure 2, top) to $15 \%$ at $10 \mathrm{~ms}$ (Figure 2, bottom). The spectra shown are scaled absolute intensities (not scaled or normalized) so that the decrease in the protonated parent intensity is due to real losses in the population of the protonated parent ion. Although the relative intensities of all ionic product vary somewhat with the instrumental tuning parameters, the decrease in the parent ion abundance as a function of detection delay is normal behavior. This is readily observed and is highly reproducible, particularly when short injection times are used and when the total ion intensity remains constant $( \pm 10 \%)$. The variation in the absolute intensities makes it difficult to discern the product ions from a few spectra. It is more useful to observe all ion intensities as a function of detection delay time. 


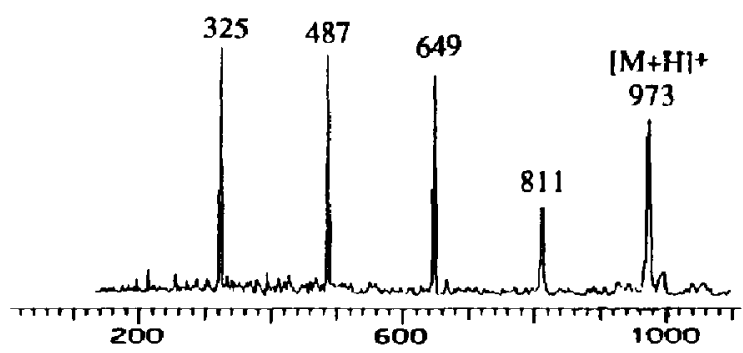

Detect Delay $=1$ millisecond

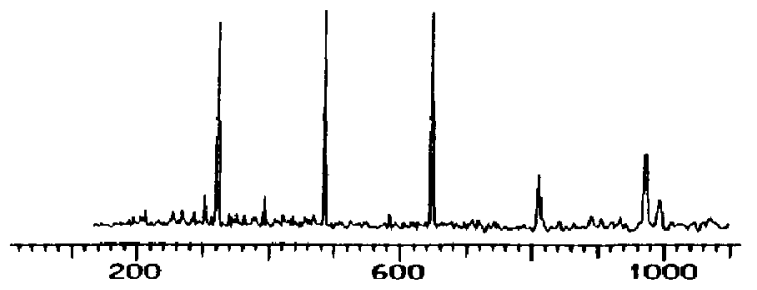

Detect Delay $=3$ milliseconds

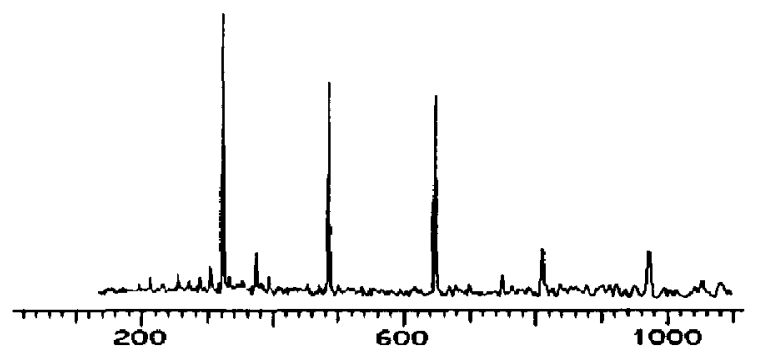

Detect Delay $=5$ milliseconds

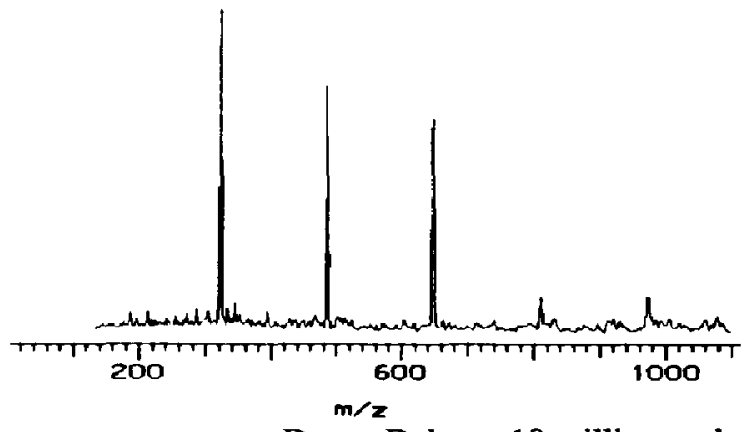

Detect Delay $=10$ milliseconds

Figure 2. Selected FAB/FTMS spectra of a-cyclodextrin with detertion delay times of $1,3,5$, and $10 \mathrm{~ms}$ after the end of the ion injection pulse. The injection period lasts $10 \mathrm{~ms}$. Spectra are produced by bombarding the sample in a glycerol:thioglycerol matrix (1:1) with a $\mathrm{Cs}^{+}$beam $(10 \mathrm{kV})$. Each spectrum uses the same absolute scaling factor so that the decrease in the abundance of $\mathrm{MH}^{+}$is due to the actual loss of the ion.
A representative plot of the absolute intensities of all ionic products as a function of detection delay time is shown in Figure 3. The decay in the intensity of the protonated parent $(m / z$ 973) is readily apparent and occurs only during the first $5 \mathrm{~ms}$. After this period, the protonated parent signal remains constant. Experiments have been performed with detection delays as long as 200 msec that show constant absolute intensities of the protonated parent beyond the initial $5 \mathrm{~ms}$. The decay in the relative intensity of the protonated parent is accompanied by a similar rise in the intensities of fragment ions corresponding to $\mathrm{m} / \mathrm{z} 325$ and 487. The ion intensity of the smaller fragment (a disaccharide fragment) rises to greater abundances than the trisaccharide fragment $(m / z$ 487). The ion intensity of the corresponding tetrasaccharide fragment $(m / z$ 649) remains essentially constant. The pentasaccharide fragment $(m / z$ 811) also decreases but not as rapidly as the protonated parent. Presumably, much of the internal energy is lost with the expulsion of a monosaccharide fragment.

Although metastable decay can be used to explain the observed phenomenon, other physical factors may operate to produce the same effect. Collisions can induce collisionally activated dissociation (CAD) or ion loss if sufficient background neutral molecules collide with the trapped ions; however, collisional processes that can account for the observed behavior can be eliminated because the background pressure during these experiments lies between high $10^{-10}$ and low $10^{-9}$ torr, yielding collision rates several orders of magnitude less than the rate of the observed decay rates (i.e., $\sim 1$ collision per second). Furthermore, CAD and collisional ejection would be more efficient with smaller molecules than larger ones, producing greater ion loss among the smaller ions.

The behavior of the total ion abundance may indicate whether an ion loss mechanism occurs. During the first $5 \mathrm{~ms}$, when the largest decrease in intensity of the protonated parent occurs, the total ion abundance remains relatively constant, with only minor statistical fluctuations $(<10 \%)$. There is a gradual decrease in the total ion abundances over a period of several hundred milliseconds that is due to either a decrease in sample concentration on the probe or actual ion loss from the analyzer cell; however, there is no behavior in the total ion population to suggest a sudden loss of a significant number of ions. These observations are consistent with a unimolecular process in which the parent ions produce smaller fragment ions, thereby maintaining the total ion population relatively constant.

Other modes of ion loss may operate with specific selectivity toward high-mass ions. For example, $z$ ejection (along the magnetic field) is dependent on the mass and radius of gyration of the ion [35-37]. The spatial distribution and translational energy of externally produced and trapped ions are not well understood and may allow several physical factors to contribute and produce the observed phenomenon. In- 


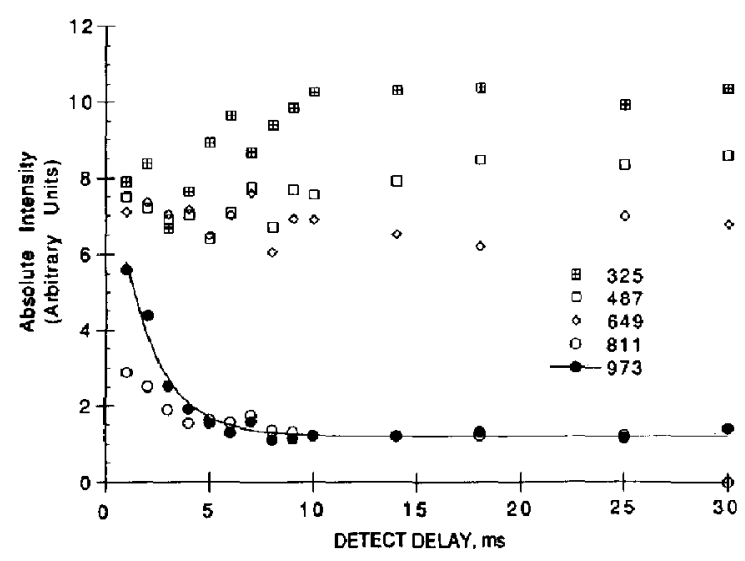

Figure 3. Representative plot of the relative intensities of all ionic products as a function of detection delay times from a period of $1-30 \mathrm{~ms}$ after the ion injection pulse. A decay in the intensity of the protonated parent $(m / z 973)$ is observed to occur up to approximately $5 \mathrm{~ms}$. Beyond this time, the ion intensity remains constant. During the same decay period, an increase in the intensities of $m / z 325$ and 487 is observed.

deed, from the plot of the ion abundances, the mass of both the protonated parent, $m / z 973$, and the $m / z 811$ fragment, decreases.

Ideally, it would be best to find a compound with a similar mass that does not undergo metastable decay to compare with the protonated parent. This is not possible because nearly all organic, and even inorgaric, ions produced by FAB contain sufficient internal energy to undergo metastable decay; however, doping the oligosaccharide solution with an alkali metal chloride allows the simultaneous formation of both alkalated and protonated parents that are found to have different decay rates. To observe the relative decomposition behavior of both species, the concentration of the dopant can be adjusted to produce nearly equal initial abundances of protonated and alkalated species. Experiments have been performed with $\mathrm{Li}^{+}, \mathrm{Na}^{+}, \mathrm{K}^{+}$, and $\mathrm{Rb}^{+}$coordinated species to observe decay rates relative to those of the protonated species. The results are a series of spectra similar to those shown in Figure 4. These spectra specifically show the competition in decay rates between coordinated species of $\mathrm{H}^{+}$and $\mathrm{K}^{+}$. The spectra are scaled to an absolute total intensity so that the losses in intensities again correspond to real losses in ion population. From the comparison of the intensities of protonated and potassiated $\alpha$-cyclodextrin (Figure 4 ), it is clear that the protonated species decrease more rapidly than the potassiated species. At $1 \mathrm{~ms}$ detection delay (Figure 4), the protonated ion is nearly $40 \%$ greater than the potassiated ion. After $3 \mathrm{~ms}$ (Figure 4), the ion intensities of both are nearly equal. At longer times, the intensities of the protonated species continue to decrease significantly faster than the potassiated species. Similar behavior is observed with all other alkali metals (i.e., $\mathrm{Li}^{+}, \mathrm{Na}^{+}$, and $\mathrm{Rb}^{+}$). In all cases, decay of the intensity of the protonated
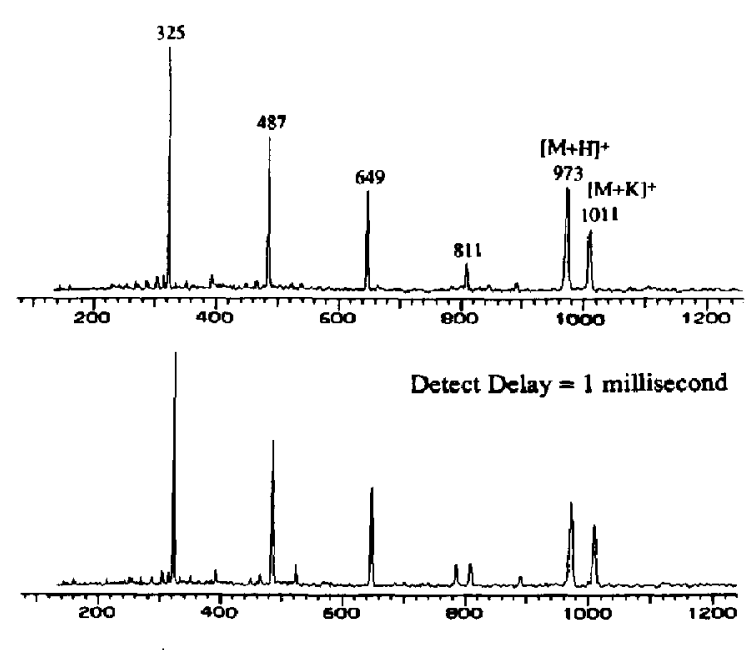

Detect Delay $=2$ milliseconds
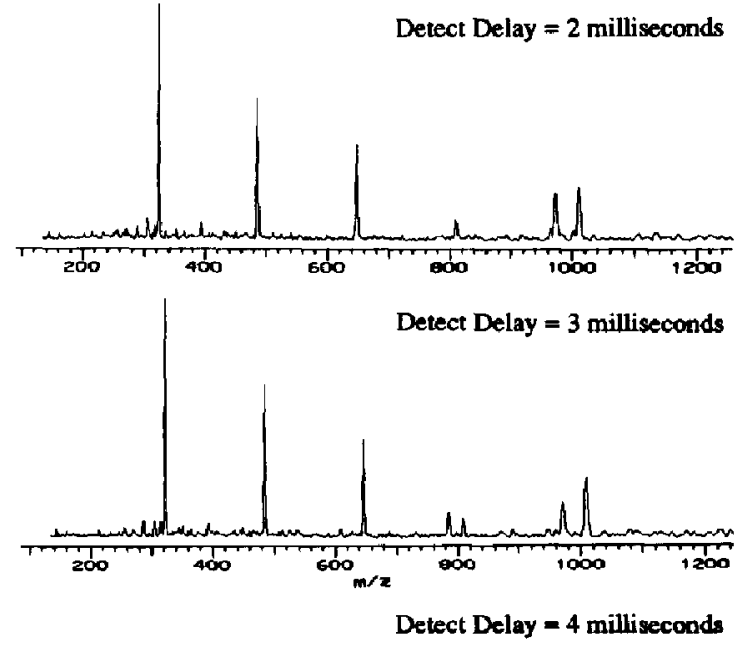

Figure 4. A series of spectra of $\alpha$-cyclodextrin doped with $\mathrm{KCl}$. Both protonated and potassiated species are observed. Fragment ions correspond only to protonated species. Lengthening the detection delay time shows a greater decay rate of the protonated species $(m / z 973)$ than the potassiated species $(m / z 1011)$. Similar results are obtained with other alkali metals, including $\mathrm{Li}, \mathrm{Na}$, and $\mathrm{Rb}$.

species is significantly faster than the decay of alkali metal coordinated species. The doped experiments are generally more difficult to perform because obtaining comparable intensities of the respective cationized parent depend strongly on the concentration of all species on the probe tip; however, once the proper concentration is obtained and maintained, the decay competition becomes highly reproducible.

The differences in metastable decay rates between protonated and alkali metal coordinated $\alpha$-cyclodextrin are consistent with the different fragmentation behavior of the two ionic species. Protonated nonderivatized oligosaccharides decompose primarily by cleavage along the glycosidic bond with little or no 
fragmentation of the glycosidic rings. Fragmentation reactions of alkali metal coordinated oligosaccharides proceed by both cleavage of the glycosidic bonds and cleavage of the glycosidic rings [38-41]. Glycosidic ring fragmentation of alkali metal coordinated oligosaccharides has been used to obtain linkage information. The decomposition of potassiated a-cyclodextrin, however, primarily produces the loss of the potassium ion. The fragment ions observed come from the protonated rather than the potassiated parent. We do observe glycosidic ring fragmentation with lithiated and sodiated oligosaccharides analyzed with FAB /FTMS.

The competition experiments rule out the possibility of selective ion loss as the primary reason for the decay in the intensity of the cationized parents. Mass selective ion loss should produce similar rates of decay in the ion abundances of both protonated and alkalated species. The relatively rapid decay of the protonated parent compared with the alkalated parent is consistent with the commonly reported observation that greater sensitivity is obtained when the alkalated molecular parents of oligosaccharides are analyzed [42]. We propose that the greater sensitivity observed with alkalated species is due to the relatively slow metastable decay rates of these species compared with the proton coordinated species.

The decay of the protonated parent intensities follows nearly complete exponential behavior. Analysis of the decay curve yields an exponential and a linear component. The abundance of the protonated parent does not totally decay to zero. Rates of metastable decay are obtained from the exponential component in Figure 2. The calculated values for the protonated compounds, averaged from 12 separate determinations over a period of 18 months, correspond to $(4.2 \pm 1.0)$ $\times 10^{2} \mathrm{~s}^{-1}$. Even with some fluctuation in the ion current, which is inherent in the FAB technique, the reproducibility of the measured rates varies by less than $20 \%$. This rate is indeed smaller than the rates of FAB-produced phenylalanine ions reported by Srhueler et al. [4].

It is important to emphasize that the metastable decay of the parent does not proceed directly to zero abundances, which would make the determination of the molecular weight difficult. There is often sufficient ion intensity to obtain high-resolution spectra of the protonated parent. Furthermore, in the design of this instrument, which is patterned after McIver's design [32], ions are accumulated so that sufficient abundances of the parent ions are obtained for the accurate determination of the molecular mass. The rapid rise of specific fragment ions (e.g., $m / z 325$ ) suggests that the product ions are produced directly from the decomposition of the parent and not from the subsequent fragmentation of larger fragment ions. The particularly large increase in the fragment ion corresponding to $\mathrm{m} / \mathrm{z} 325$ may be inferred as a particularly stable characteristic of the disaccharide units because, presum- ably, all glycosidic bonds in this molecule are identical.

The nature of the vibrational excitation during $F A B$ ionization is not clear. The FAB ionization process is still not completely understood, although it is apparent that the process involves several steps [25, 26, 43-45]. A study by Schueler et al. [4] to determine the effects of varying the primary beam energy from 3 to $13 \mathrm{keV}$ on the unimolecular rate constants shows no dependence between the two. Similar experiments were attempted with FTMS, but the overall signal-to-noise ratio varied greatly with the primary beam energy. It has also been shown that the degree of vibrational excitation during $\mathrm{FAB}$ ionization depends on the length of time that the ions remain in the selvedge region or in the quasi-gas state [46]. In any case, the weaker abundances of fragment ions in FAB ionization, specifically for oligosaccharides, are due to the fact that FAB is generally a softer ionization technique and that the larger molecules that are often analyzed have significantly slower decomposition rates. Slow metastable decay has several important analytical implications. It means that the degree of fragmentation can be manipulated without using collisional or other activation methods. The slow rates also account for the differences in the degree of fragmentation in spectra obtained between sector and FTMS instruments.

\section{Acknowledgment}

Funds provided by the University of California Cancer Research Coordinating Committee, and the University of California, Davis, Commiltet on Researth, are gratelully ackıowledged.

\section{References}

1. Boesl, U.; Neusser, H. J.; Weinkauf, R.; Schlag, E. W. J. Phys. Chem. 1982, 86, 4857.

2. Bunker, D. L.; Wang, F.-M. J. Am. Chem. Soc. 1977, 99, 7457.

3. Kofel, P.; McMahon, T. B. J. Phys. Chem. 1988, 92, 6174.

4. Schueler, B.; Beavis, R.; Ens, W.; Main, D. E.; Tang, X; Standing, K. G. Int. J. Mass Spectrom. Ion Pracesses 1989, 92, 185.

5. Neusser, H. J. Int. I. Mass Spectrom. Ion Processes 1987, 79, 141.

6. Beyiun, J. H.; Funtainie, A. E.; Lester, G. R. Int. I. Muss Spectrom. Ion Phys. 1972, 8, 341.

7. Kim, Y. H.; Kim, M. S. Rapid Commun. Mass Spectrom. 1991, $5,25$.

8. Kinsel, G. R.; Johnston, M. V. Anal. Chem. 1988, 60, 2084.

9. Knewstubb, P. F.; Reid, N. W. Int. J. Mass Spectrom. Ion Phys. $1971,5,361$.

10. Kühlewind, H.; Kiermeier, A.; Neusser, H. J.; Schlag, E. W. J. Chem. Phys. 1987, 87, 6488.

11. Rockney, B. H.; Grant, E. R. J. Chem. Phys. 1983, 79, 708.

12. For reviews, see Robinson, P. J.; Holbrook, K. A. Unimolecular Reactions; Wiley: New York, 1972; Forst, W. Theory of Unimolecular Reactions; Academic Press: New York, 1973.

13. Demirev, P.; Olthoff, J. K.; Fenselau, C.; Cotter, R. J. Anal. Chem. 1987, 59, 1951.

14. Egsgaard, H.; Carlsen, L. Chem. Phys. Lett. 1988, 147, 30.

15. Favretto, D.; Traldi, P.; Bravo, P.; Viani, F. Rapid Commun. Mass Spectrom. 1991, 5, 72. 
16. Futrell, J. H,; Stephan, K.; Märk, T. D. J. Chem. Phys. 1982, 76, 5893.

17. Gordon, S. M.; Krige, G. J.; Reid, N. W. Int. J. Mass Spectrom Ion Phys. 1975, 14, 109.

18. Huntress, W. T. J.; Sen, S. D. K.; Jennings, K. R.; Bowers, M. T. Int. I. Mass Spectrom. Ion Phys. 1977, 24, 25.

19. Kiermeier, A.; Ernstberger, B.; Neusser, H. J.; Schlag, E. W. Ber. Bunsenges. Phys. Chem. 1988, 92, 437.

20. Kühlewind, H.; Kiermeier, A.; Neusser, H. J. J. Chem. Phys. $1986,85,4427$.

21. Thorne, G. C.; Ballard, K. D.; Gaskell, S. J. J. Am. Soc. Mass Spectrom. 1990, 1, 249

22. Wankenne, H.; Caprace, G.; Momigny, J. Int. J. Mass Spectrom. Ion Processes 1984, 57, 149.

23. King, B.; Ziv, A.; Lin, S.; Tsong, I. J Chem. Phys. 1985, 82, 3641.

24. Craig, A.; Detrick, P. I. Am. Chem. Soc. 1985, 107, 6707.

25. Barber, M.; Bordoli, R. S.; Sedgwick, R. D; Tyler, A. N. 7 . Chem. Soc. Chem. Commun. 1981, 325.

26. Barber, M.; Bordoli, R. S.; Sedgwick, R. D.; Vickerman, J. J. Chem. Soc., Faraday Trans. 1 1982, 78, 1291.

27. Guan, S.; Mclver, R. T. Ir. Proceedings of the 38th ASMS Conference on Mass Spectrometry and Allied Topics; Tucson, AZ, June, 1990.

28. Barlow, S. E.; Dunn, G. H.; Schauer, M. Phys, Ret. Lett, 1984, $52,902$.

29. Carroll, J. A.; Ngoka, L.; McCullough, S. M.; Gard, E.; Jones, A. D.; Lebrilla, C. B. Anal. Chem. 1991, 63, 2526.
30. Hunt, D. F.; Shabanowitz., J.; Yates, J.R. III. J. Chem. Soc. Chem. Commur. 1987, 548.

31. Molver, R. T. Jr; Hunter, R. L.; Bowers, W. D. Int. I. Mnss Spectrom. Ion Processes 1985, 64, 67.

32. Lebrilia, C. B.; Wang, D. T. S.; Mizoguchi, T. J.; Mclver, R. T. Jr. J. Am. Chem. Soc. 1989, 111, 8593.

33. McCullough, S. M.; Gard, E.; Lebrilla, C. B. Int. J. Mass Spectrom. lon Processes 1991, 107, 91.

34. Hunt, D. F.; Shabanowitz, J.; Yates, J. R. III; Mclver, R. T. Jr.; Hunter, R. L.; Syka, J. E. P. Artal. Chem. 1985, 57, 2728.

35. Caravatti, P.; Allemann, M. Org. Mass Spectrom. 1991, 26, 514.

36. Hanson, C. D.; Castro, M. E.; Kerley, E. L.; Russell, D. H. Anal. Chem. 1990, 62, 520 .

37. Wang, M.j Marshall, A. G. Anal. Chem. 1990, 62, 515.

38. Hofmeister, G. E.; Zhou, Z.; Leary, J. A. I. Am. Chem. Soc. 1991, 113, 5964.

39. Zhou, Z.; Ogden, S.; Leary, J. A. I. Org. Chem. 1990, 55, 5444.

40. Spengler, B.; Dolce, J. W.; Cotter, R. J. Anal. Chem. 1990, 62, 1731.

41. Orlando, R.; Bush, C. A.; Fenselau, C. Biomed. Environ. Mass Spectrom. 1990, 19, 747.

42. Dell, A. Adv. Carbohydr. Chem. Biochem. 1987, 45, 19.

43. Fenselau, C.; Cotter, R. J. Chem. Rev. 1987, 87, 501.

44. Pachuta, S. J.; Cooks, R. G. Chem. Rev. 1987, 87, 647.

45. Williams, D. H.; Findeis, A. F.; Naylor, S.; Gibson, B. W. J. Am. Chem. Soc. 1987, 109, 1980.

46. Sunner, J.; Morales, A.; Kebarle, P. Anal. Chem. 1988, 60, 98. 